\title{
Index rerum ad Vol. 174
}

\section{Cotfecit: C. L·oeb-Schürch}

(B) $=$ Book Reviews - Buchbesprechungen -Livres nouveaux

Ablatio, v. Anterior cerclage, Encircling band,

Polyglycolic acid, Retinal detachment Abrégé d'ophtalmologie 299 (B) Acrylic implants, v.

Reconstruction Acuité visuelle, v. Cavernous degeneration, Dys-

thyroid neuropathy, Retinitis pigmentosa Adaptation à Гobscurité, v. Cone disease, Dark adaptation, Retinitis pigmentosa Adenoma, v. Epithelial tumour Aderhaut, v. Iris injuries, Larva migrans,

Solitärgranulom Adhérences vitréo-rétiniennes, v. Gitterfiguren Ageing, v. Cavernous degeneration, Optic disc Altersveränderungen, v. Cavernous degeneration,

Optic disc Anetoderma; Jadassohn-type anetoderma in association with keratoconous and cataract 181 Angiographie fluoresceïnique, v. Calcium dobe-

silate, Iris masses, Larva migrans lentis,

Retinitis pigmentosa. Vacuum extractor Angle camérulaire, v. Corticosteroid glaucoma, Filtration angle, Syphilitic keratitis, Trabekel-

system. Twins Aniridia, v. Wilms' tumour Aniseikonie, v. Aphakie Anniversaire; pour le 70 anniversaire du pro-

fesseur Jules Francois 346 Anomalie pupillaire, v. Parinaud-Syndrom,

Ultrastructure Anterior cerclage and accessory implants for

post-equatorial breaks 274 Antibiotics, v. Prophylactic antibiotics Anticorps, v. Iris injuries

Aphakia, v. Pupillary block Aphakie; einseitige Aphakie - konventionelle

Kontaktlinse und Aniseikonie 340 Aqueous humor, v. Filtration angle, Trabekel-

system Argon laser, v. Pupillary block Argyll-Robertson Phänomen, v. Parinaud-Syndrom

Arteriitis temporalis, v. Pseudopapillitis vascularis

Atrophie irienne, v. Iris atrophy

Atrophie optique, v. Dysthyroid neuropathy,

Syphilitic keratitis Augenbinnendruck, v. Corticosteroid glaucoma.

Encircling band, Expulsive hemorrhage,

Pupillary block, Syphilitic keratitis Augenkomplikationen, v. Anterior cerclage,

Expulsive hemorrhage, Retinal detachment,

Vacuum extractor Augenmuskeln, v. Extrinsic paralysis, Ocular

malformations, Parinaud-Syndrom Autoimmunkrankheit, v. Iris injuries Autonomous nervous

system, v. Riley-Day

syndrome

Bandes en grille, v. Gitterfiguren

Bâtonnets, v. Cone disease

Bengal-Rosa-Vitalfärbung, v. Trockenes Auge

Bestrahlung, v. Orbital development, Pterygium 
Bindehaut, v. Dermolipoma, Kérato-conjonctivite, Pterygium, Riley-Day syndrome, Temporal pterygium, Trockenes Auge

Binokularsehen, v. Aphakie

Biochemie, v. Corticosteroid glaucoma, Myotonic dystrophy

Biometrie, v. Orbital development

Biomicroscopie, v. Larva migrans lentis, Vésicule libre

Blicklähmung, v. Extrinsic paralysis, Parinaud-Syndrom

Blutung, v. Calcium dobesilate, Expulsive hemorrhage

Book reviews 179 (B), 240 (B), 299 (B)

Buchbesprechungen 179(B), 240(B), 299(B)

Calcium dobesilate; assessment of calcium dobesilate in diabetic retinopathy. A double-blind

clinical investigation 47

Carcinoma, v. Epibulbar carcinoma, Mucoepi-dermoid carcinomas

Cataract, v. Anetoderma, Expulsive hemorrhage, Myotonic dystrophy, Prophylactic antibiotics

Cavernous degeneration; 14 cases of cavernous degenerations of the optic nerve 316

Cerclage, v. Anterior cerclage

348

Index rerum ad Vol. 174

Chamber angle, v. Corticosteroid glaucoma,

Filtration, angle, Syphilitic keratitis, Trabekel-system, Twins

Champ visuel, v. Chiasm tumors, Retinitis pig-mentosa

Chiasm tumors; differential diagnostic aspects between optic neuritis and chiasm tumors 106

Chirurgie, v. Anterior cerclage, Dysthyroid neuropathy, Encircling band, Expulsive hemorrhage,

Polyglycolic acid, Prophylactic antibiotics, Pterygium, Reconstruction, Retinal detachment

Chorioidea, v. Iris injuries, Larva migrans, Solitärgranulom

Choristoma, v. Dermolipoma

Choristoma osseum orbitae. A clinlcopathological case report 176

Choroidopathy, v. Encircling band

Choroïdose myopique, v. Cone disease

Chromosom, v. Wilms' tumour

Ciliary body, v. Epithelial tumour, Filtration angle

Climat, v. Pterygium

Clinicopathological case reports 55, 171

Cobaye, v. Iris injuries

Color vision, v. Cone disease, Retinitis pigmentosa

Colour vision deficiencies III 240 (B)

Complications oculaires, v. Anterior cerclage, Expulsive hemorrhage. Retinal detachment.

Vacuum extractor

Computer, v. Optic disc, Trockenes Auge

Cone disease; peripheral cone disease 145

Congenital glaucoma, v. Twins

Congenital syphilis, v. Syphilitic keratitis

Conjonctivites; les conjonctivites à virus 299 (B)

Conjunctiva, v. Dermolipoma, Kérato-conjoncti-vite, Pterygium, Riley-Day syndrome, Temporal pterygium, Trockenes Auge 
Contact lens, v. Aphakie

Cornea, v. Anetoderma, Dystrophie tachetée, Kérato-conjonctivite, Pterygium, Riley-Day

syndrome, Syphilitic keratitis, Temporal pterygium, Trockenes Auge, Twins

Corps ciliaire, v. Epithelial tumour, Filtration angle

Corps vitré, v. Gitterfiguren, Vésicule libre

Corticosteroid glaucoma 195

Corticosteroids, v. Dysthyroid neuropathy

Cristallin, v. Anetoderma, Larva migrans, Prophylactic antibiotics

Cyst, v. Iris masses, Vésicule libre

Cytology, v. Corticosteroid glaucoma, Dystrophie tachetée

Dark adaptation, v. Cone disease, Retinitis pigmentosa

Dark adaptation in diabetics 322

Déchirures rétiniennes, v. Anterior cerclage, Encircling band, Polyglycolic acid, Retinal

detachment

Décollement rétinien, v. Anterior cerclage, Encircling band, Polyglycolic acid. Retinal

detachment

Decompression, v. Dysthyroid neuropathy

Dégénérescence caverneuse, v. Cavernous degeneration

Dendritic lines, v. Gitterfiguren

Dermatologie, v. Anetoderma

Dermolipoma; epibulbar osteoma combined with dermolipoma 58

Développement orbitaire, v. Orbital development

Diabetes mellitus, v. Calcium dobesilate, Dark adaptation, Ultrastructure

Diabetic retinopathy, v. Calcium dobesilate, Dark adaptation

Differentialdiagnose, v. Chiasm tumors, Iris masses, Solitärgranulom

Differentialdiagnose der Augenkrankheiten 240 (B)

Dobesilate de calcium, v. Calcium dobesilate

Documenta Ophthalmologica. Xth ISCERG Symposium 179 (B)

Dunkeladaptation, v. Cone disease, Dark adaptation, Retinitis pigmentosa

Dysthyroid neuropathy; management of dysthyroid optic neuropathy 327

Dystrophie myotonique, v. Myotonic dystrophy

Dystrophie tachetée; etude au microscope à ba-layage des cultures de la dystrophie tachetée de la cornée 75

Elektromyogramm, v. Extrinsic paralysis

Elektronenmikroskopie, v. Epibulbar carcinoma. Filtration angle, Retinoblastoma, Tubuläre

Inklusionen, Ultrastrucíure

Elektrookulogramm, v. Cone disease

Elektroretinogramm, v. Cone disease, Retinitis pigmentosa

Embolie, v. Vacuum extractor

Embryologie, v. Gitterfiguren, Ocular malformations, Wilms' tumour

EMG, v. Extrinsic paralysis

Encircling band; the encircling silastic $3 \mathrm{~mm}$ band without cautery in retinal detachment surgery 336

Index rerum ad Vol. 174

349 
Endocrinology v. Dysthyroid neuropathy Endothelien, v. Tubuläre Inklusionen Enucleation, v. Orbital development Enzyme, v. Corticosteroid glaucoma, Myotonic dystrophy EOG, v. Cone disease Epibulbar carcinoma; metastatic epibulbar carcinoma 251 Epibulbäres Osteom, v. Dermolipoma Epithelial tumour (adenoma) of the ciliary body

and iris 55 Epithelium pigmentaire, v. Gitterfiguren, Retinitis

pigmentosa ERG v. Cone disease, Retinitis pigmentosa Excavation glaucomateuse, v. Syphilitic keratitis Exophthalmos, v. Dysthyroid neuropathy Experimentelle Forschung, v. Corticosteroid glaucoma, Dystrophie tachetée, Filtration angle, Iris injuries, Myotonic dystrophy, Retinoblastoma, Trabekelsystem, Vacuum extractor Expulsive hemorrhage; some observations concerning expulsive hemorrhage 100 Extrinsic paralysis; histologie and electromyo-graphic research on a case of recurrent alternating extrinsic paralysis 40

Farbsehen, v. Cone disease, Retinitis pigmentosa

Farbsinnstörungen; die Farbsinnstòrungen und ihre Prüfung in der Praxis 180 (B)

Filtration angle; experimental morphological study on structure and function of the filtration angle of the rat eye 285

Fleckförmige Hornhautdystrophie, v. Dystrophie tachetée

Fluoroangiographie, v. Calcium dobesilate, Iris masses, Larva migrans lentis, Retinitis pigmentosa, Vacuum extractor

Gefässe, v. Calcium dobesilate, Cavernous degeneration, Encircling band, Expulsive

hemorrhage, Gitterfiguren, Iris masses, Pseudopapillitis, Tubuläre Inklusionen, Vacuum extractor

Genetics, v. Myotonic dystrophy, Riley-Day syndrome, Twins, Wilms' tumour

Gesichtsfeld, v. Chiasm tumors, Retinitis pigmentosa

Gitterfiguren bzw. dendritische Streifen der Netz-hautperipherie 2

Glandes de Moll. v. Mucoepidermoid carcinomas

Glaskörper, v. Gitterfiguren, Vesicule libre

Glaskòrperretraktion. v. Anterior cerclage

Glaucoma, v. Cavernous degeneration, Corticosteroid glaucoma, Expulsive hemorrhage, Iris atrophy, Syphilitic keratitis. Twins

r-Glutamyl transpeptidase, v. Myotonic dystrophy

Glutathion, v. Myotonic dystrophy

Gonioscopy, v. Syphilitic keratitis

Graft, v. Reconstruction

Guinea pig, v. Iris injuries

Haemorrhagie, v. Calcium dobesilate. Expulsive hemorrhage

Helminthiasis, v. Larva migrans lentis, Solitär-granulom

Hérédité, v. Myotonic dystrophy, Riley-Day syndrome. Twins, Wilms' tumour

Hernie cérébrale, v. Parinaud-Syndrom

Hirnhernie, v. Parinaud-Syndrom

Hirnödem, v. Parinaud-Syndrom

Histochemie, v. Corticosteroid glaucoma

Histogenesis, v. Retinoblastoma

Histologie, v. Anetoderma, Cavernous degeneration, Corticosteroid glaucoma, Choristoma osseum, Dermolipoma, Epibulbar carcinoma, Epithelial tumour, Extrinsic paralysis, Filtration 
angle, Gitterfiguren, iris atrophy, Mucoepidermoid carcinomas, Pseudopapillitis, Retinoblastoma, Riley-Day syndrome, Tubuläre Inklusionen

Hormones sexuelles, v. Trockenes Auge

Hornhaut, v. Anetoderma, Dystrophie tachetée, Kérato-conjonctivite, Pterygium, Riley-Day

syndrome. Syphilitic keratitis. Temporal pterygium, Trockenes Auge, Twins

Humeur aqueuse, v. Filtration angle, Trabekelsystem

Hydrocephalus, v. Parinaud-Syndrom

Hydrodynamik, v. Trabekelsystem

Hyperperméabilité vasculaire, v. Calcium dobesilate

Hypertension, v. Expulsive hemorrhage

Hyperthyroidism, v. Dysthyroid neuropathy

Implantation, v. Anterior cerclage, Iris injuries,

Reconstruction Innere Erkrankungen und Auge 240 (B) Intraocular tension, v. Corticosteroid glaucoma,

Encircling band. Expulsive hemorrhage,

Pupillary block. Syphilitic keratitis Iris, v. Epithelial tumour

350

Index rerum ad Vol. 174

Iris atrophy; patchy iris atrophy in a case of

chronic simple glaucoma 92 Iris injuries; response to experimental implantation

of autologous iris tissue and to iris injuries 158 Iris masses; fluorescein angiographic behaviour of

iris masses 217 Irismuskel, v. Ultrastructure Irradiation, v. Orbital development, Pterygium

Jumeaux, v. Twins

Kalziumdobesilat, v. Calcium dobesilate

Kammerwasser, v. Filtration angle, Trabekel-system

Kammerwinkel, v. Corticosteroid glaucoma, Filtration angle, Syphilitic keratitis, Trabekel-

system, Twins

Kaninchen, v. Corticosteroid glaucoma, Iris injuries, Photoreceptors, Vacuum extractor

Katarakt, v. Anetoderma, Expulsive hemorrhage, Myotonic dystrophy, Prophylactic antibiotics

Keratitis, v. Syphilitic keratitis

Kérato-conjonctivite; la kérato-conjonctivite de Tadéno-virus type 1061

Keratoconjunctivitis sicca, v. Trockenes Auge

Keratoconus, v. Anetoderma

Keratozyt, v. Dystrophie tachetee

Klima, v. Pterygium

Kontaktlinse, v. Aphakie

Kunststoffharz, v. Reconstruction

Kyste, v. Iris masses, Vésicule libre

Lacrimation, v. Riley-Day syndrome, Trockenes Auge

Lähmung, v. Extrinsic paralysis

Lamina-Test, v. Solitärgranulom

Lapin, v. Corticosteroid glaucoma, Iris injuries, Photoreceptors, Vacuum extractor

Larva migrans, v. Solitärgranulom

Larva migrans lentis 14 
Laser, v. Pupillary block

Latticed lines, v. Gitterfiguren

Lens, v. Anetoderma, Larva migrans, Prophylactic antibiotics

Lentille de contact, v. Aphakie

Leukoma corneae, v. Riley-Day syndrome

Lid, v. Mucoepidermoíd carcinomas

Linse, v. Anetoderma, Larva migrans, Prophylactic antibiotics

Lipodermoid, v. Dermolipoma

Livres nouveaux 179 (B), 240 (B), 299 (B) Lysosome, v. Corticosteroid glaucoma

Macula, v. Retinitis pigmentosa

Macular corneal dystrophy, v. Dystrophie tachetee

Malformation, v. Ocular malformations, Vésicule

libre, Wilms' tumour Medikamentöse Augentherapie. Grundlagen und

Praxis 179 (B) Meerschweinchen, v. Iris injuries Melanoblastoma, v. Tubuläre Inklusionen

Metabolism, v. Calcium dobesilate, Myotonic

dystrophy, Riley-Day syndrome Metastase, v. Epibulbar carcinoma Methode, v. Anterior

cerclage, Encircling band,

Polyglycolic acid, Pterygium, Pupillary block,

Reconstruction Microphthalmia, v. Ocular malformations Microscopie electronique, v. Epibulbar carcinoma,

Filtration angle, Retinoblastoma, Tubuläre

Inklusionen, Ultrastructure Microscopie electronique à balayage, v. Dystrophie

tachetee, Photoreceptors Migration du pigment, v. Iris atrophy Mikropräzipitationstest, v.

Solitärgranulom Mintezol, v. Larva migrans lentis, Solitärgranulom Minzolum, v. Larva migrans lentis, Solitärgranulom Mollsche Drüsen, v. Mucoepidermoid carcinomas Mucoepidermoid carcinomas of the lid. A clinico-

pathologic report of two cases 171 Mucopolysaccharide, v. Corticosteroid glaucoma Muscle

irien, v. Ultrastructure Muscles oculaires, v. Extrinsic paralysis, Ocular

malformations, Parinaud-Syndrom Myopathie, v. Extrinsic paralysis Myopia, v. Cavernous

degeneration, Cone

disease Myotonic dystrophy; cataract and j'-glutamyl cycle

in myotonic dystrophy 167

Nematoden, v. Larva migrans lentis, Solitärgranulom

Nephroblastoma, v. Wilms' tumour

Nerve terminal, v. Ultrastructure

Nervenendigung, v. Ultrastructure

Netzhaut, v. Anterior cerclage, Calcium dobesilate. Encircling band, Gitterfiguren, Polyglycolic acid, Retinal detachment, Retinitis pigmentosa, Retinoblastoma, Solitärgranulom

Index rerum ad Vol. 174

35]

Neuritis optica, v. Chiasm tumors

Neuro-Ophthalmologie, v. Chiasm tumors. Parinaud-Syndrom, Riley-Day syndrome

Neuro-ophthalmology; symposium on neuro-ophthalmology. Transactions of the New Orleans

Academy of Ophthalmology 179 (B)

Nystagmus, v. Cone disease 
Ocular malformations; frequent coexistence of ocular malformations in externally malformed human fetuses 241

Ocular muscles, v. Extrinsic paralysis, Ocular malformations, Parinaud-Syndrom

Ocular therapy 180 (B)

CEdème cerebral, v. Parinaud-Syndrom

(Eil sec, v. Trockenes Auge

Operation, v. Anterior cerclage, Dysthyroid neuropathy. Encircling band, Expulsive hemorrhage,

Polyglycolic acid, Prophylactic antibiotics, Pterygium, Reconstruction, Retinal detachment

Ophtalmopathie dysthyroïdienne, v. Dysthyroid neuropathy

Ophthalmodynamographie 240 (B)

Ophthalmoplegia, v. Extrinsic paralysis, Parinaud-Syndrom

Ophthalmoskopie 180 (B)

Optic atrophy, v. Dysthyroid neuropathy. Syphilitic keratitis

Optic disc; surface and shape of the optic disc in healthy subjects in various age groups.

Application of computer picture processing 261

Optic neuritis, v. Chiasm tumors

Opticus, v. Cavernous degeneration, Chiasm tumors, Dysthyroid neuropathy. Optic disc,

Pseudopapillitis

Orbita, v. Choristoma osseum, Reconstruction

Orbital development; effects of radiation after enucleation without implantation on orbital

development of patients with retinoblastoma 137

Ordinateur, v. Optic disc, Trockenes Auge

Ostéome épibulbaire, v. Dermolipoma

Pädo-Ophthalmologie, v. Choristoma osseum, Orbital development, Solitärgranulom

Papule, v. Cavernous degeneration, Dysthyroid neuropathy, Optic disc, Pseudopapillitis

Paralysis, v. Extrinsic paralysis

Parasiten, v. Larva migrans lentis, Solitärgranulom

Parasympathicus, v. Riley-Day syndrome

Parese, v. Parinaud-Syndrom

Parinaud-Syndrom; das Parinaud-Syndrom als Fernzeichen bei supra- und infratentoriellen

raumfordernden Prozessen Ill

Paupière, v. Mucoepidermoid carcinomas

Perimetrie, v. Chiasm tumors, Retinitis pigmentosa

Photoreceptor optics 299 (B)

Photoreceptors; scanning electron microscopy of photoreceptors 280

Photorezeptoren, v. Cone disease, Retinitis pigmentosa

Pig, v. Corticosteroid glaucoma

Pigmentepithel, v. Gitterfiguren, Retinitis pigmentosa

Pigmentmigration, v. Iris atrophy

Pinguecula, v. Pterygium

Plastische Chirurgie des Kopf- und Halsbereichs und der weiblichen Brust 299 (B)

Polyglycolic acid suture in retinal detachment surgery 277

Pore, v. Corticosteroid glaucoma

Prophylactic antibiotics in cataract surgery 52

Prothese, v. Reconstruction

Pseudopapillitis vascularis; statistical study of pseudopapillitis vascularis 266 
Pterygium, v. Temporal pterygium

Pterygium im Qatar 81

Pupillary block; management of aphakic pupillary block. A new argon-laser approach 310

Pupillenanomalie, v. Parinaud-Syndrom, Ultra-structure

Rabbit, v. Corticosteroid glaucoma. Iris injuries, Photoreceptors, Vacuum extractor

Radiation, v. Orbital development, Pterygium

Raster-Elektronenmikroskopie, v. Dystrophie tachetée, Photoreceptors

Rat, v. Filtration angle

Recherches expérimentales, v. Corticosteroid

glaucoma, Dystrophie tachetée, Filtration angle, Iris injuries, Myotonic dystrophy,

Retinoblastoma, Trabekelsystem, Vacuum extractor

Rechute, v. Retinal detachment

Reconstruction of the orbit with antibiotic acrylic in cases of severe bone loss 301

Relapse, v. Retinal detachment

Résine acrylique, v. Reconstruction

Retina, v. Anterior cerclage, Calcium dobesilate. Encircling band, Gitterfiguren, Polyglycolic

acid, Retinal detachment, Retinitis pigmentosa, Retinoblastoma, Solitärgranulom

352

Index rerum ad Vol. 174

Retinal detachment, v. Anterior cerclage. Encircling

band, Polyglycolic acid Retinal detachment; clinical survey of the forms,

number and localization of retinal tears in

cases of relapses and recurrences in retinal

detachment 210 Retinitis pigmentosa; functional evaluation in

central retinitis pigmentosa 121 Retinoblastoma; the histogenesis of retinoblastoma.

An electron-microscopic analysis of rosette 129 Réínopathie diabétique, v. Calcium dobesilate,

Dark adaptation Retinoscopy: a decision-oriented manual of

retinoscopy 179 (B) Retraction vitréenne, v. Anterior cerclage Rezidiv, v. Retinal detachment

Riley-Day syndrome; the Riley-Day syndrome.

Familial dysautonomy, central autonomic

dysfunction 20 Risse der Netzhaut, v. Anterior cerclage. Encircling band, Polyglycolic acid,

Retinal

detachment Rods, v. Cone disease Röntgen-Untersuchung, v. Chiasm tumors, Orbital

development, Parinaud-Syndrom Rosette, v. Retinoblastoma

Scanning electron microscopy, v. Dystrophie

tachetée, Photoreceptors Schirmer Test, v. Riley-Day syndrome Schlemm's canal, v.

Corticosteroid glaucoma,

Filtration angle Schwein, v. Corticosteroid glaucoma Scleral buckling, v. Anterior cerclage,

Encircling

band Sclérose vasculaire, v. Cavernous degeneration,

Expulsive hemorrhage, Pseudopapillitis Scotoma, v. Dysthyroid neuropathy, Retinitis

pigmentosa Sehschärfe, v. Cavernous degeneration, Dysthyroid

neuropathy, Retinitis pigmentosa Sekundärglaukom, v. Corticosteroid glaucoma.

Syphilitic keratitis Sexualhormone, v. Trockenes Auge Sklera, v. Pterygium Solitärgranulom, v.

Larva migrans lentis Solitärgranulom; über das Solitärgranulom bei 
okularer Toxocí/ra-cö«/.v-lnfektion (Hundespulwurm) 10 Stäbchen, v. Cone disease

Statistik, v. Pseudopapillitis, Trockenes Auge Subchoroidal hemorrhage, v. Expulsive hemorrhage Surgery, v. Anterior cerclage, Dysthyroid neuropathy. Encircling band. Expulsive hemorrhage. Polyglycolic acid, Prophylactic antibiotics, Pterygium, Reconstruction, Retinal detachment Surgery of the iris and ciliary body 300 (B) Syndrome, v. Parinaud-Syndrom, RileyDay syndrome, Wilms' tumour Synechia, v. Pupillary block, Syphilitic keratitis Syphilitic keratitis; secondary glaucoma due to inactive congenital syphilitic interstitial keratitis 188 Système nerveux autonome, v. Riley-Day syndrome

Tears, v. Riley-Day syndrome, Trockenes Auge

Tears of retina, v. Anterior cerclage, Encircling band, Polyglycolic acid. Retinal detachment

Temporal pterygium; is the occurrence of a temporal pterygium really so rare? 88

Temporalisdynamographie; Grundlagen und Methodik der Ophthalmodynamographie, Ophthalmodynamometrie. Temporalisdynamographie 300 (B)

Tension oculaire, v. Corticosteroid glaucoma. Encircling band, Expulsive hemorrhage. Pupillary block, Syphilitic keratitis

Terminaison nerveuse, v. Ultrastructure

Therapie, v. Calcium dobesilate, Dysthyroid neuropathy. Encircling band, Prophylactic

antibiotics, Pseudopapillitis, Solitärgranulom, Syphilitic keratitis

Thyreoidea, v. Dysthyroid neuropathy

Tonographie, v. Syphilitic keratitis

Toxocara canis, v. Larva migrans lentis. Solitärgranulom

Toxoplasmose, v. Solitärgranulom

Trabeculum corneosclerale, v. Corticosteroid glaucoma, Filtration angle

Trabekelsystem; die Rolle des Trabekelsystems beim Kammerwasserwechsel aufgrund von

Modellversuchen 151

Tränen, v. Riley-Day syndrome, Trockenes Auge

Transplantation, v. Reconstruction

Trockenes Auge; statistischer Beitrag zur Sym-ptomatik des trockenen Auges 65

Tubuläre Inklusionen und virusähnliche Pariikeln in intraokularen Melanoblastomen 255

Tumor, v. Chiasm tumors, Choristoma osseum, Dermolipoma, Epibulbar carcinoma, Epithelial Index rerum ad Vol. 174

353

tumour, Iris masses, Mucoepidermoid carcinomas, Orbital development, Parinaud-Syndrom, Retinoblastoma, Wilms' tumour Twins; congenital glaucoma in only one of identical twins 185 Ultrastructure of iris muscle in diabetes mellitus

228 Uveitis, v. Iris injuries

Vacuum extractor; retina hemodynamic modifications after experimental application of the vacuum extractor 224

Vaisseaux, v. Calcium dobesilate, Cavernous degeneration, Encircling band, Expulsive hemorrhage, Gitterfiguren, Iris masses, Pseudo-papillitis, Tubuläre Inklusionen, Vacuum extractor

Varia 180

Vererbung, v. Myotonic dystrophy, Riley-Day syndrome, Twins, Wilms' tumour 
Vésicule libre d'aspect kystique du vitré. Etude biomicroscopique - Discussion diagnostique et pathogénique 96

Vessels, v. Calcium dobesilate, Cavernous degeneration, Encircling band, Expulsive hemorrhage, Gitterfiguren, Iris masses, Pseudopapillitis, Tubuläre Inklusionen, Vacuum extractor

Vieillissement, v. Cavernous degeneration, Optic

disc Virus, v. Kérato-conjonctivite Virus-like particles, v. Tubuläre Inklusionen Vision des couleurs, v. Cone disease, Retinitis

pigmentosa Visual acuity, v. Cavernous degeneration, Dys-

thyroid neuropathy, Retinitis pigmentosa Visual field, v. Retinitis pigmentosa Visually evoked cortical potentials, v. Chiasm

tumors Vitreoretinale Adhärenzen, v. Gitterfiguren Vitreous body, v. Gitterfiguren, Vésicule libre Vitreous retraction, $v$. Anterior cerclage

Wachstum der Orbita, v. Orbital development Wilms' tumour; aniridia - Wilms' tumour syndrome

35

X-ray examination, v. Chiasm tumors, Orbital development, Parinaud-Syndrom

Zapfen, v. Cone disease

Z. Ziliarkörper

v. Epithelial tumour. Filtration angle Zwillinge

v. Twins Zyste

v. Iris masses

Vésicule libre Zytologie

v. Corticosteroid glaucoma

Dystrophie tachetée 